\title{
The conditions in the existence results for discontinuous games by Reny and by Simon and Zame are incomparable*
}

\author{
Guilherme Carmona $^{\dagger} \quad$ Konrad Podczeck ${ }^{\ddagger}$ \\ University of Surrey Universität Wien
}

May 10, 2018

\begin{abstract}
A natural way to relate the existence theorems of Reny (1999) and Simon and Zame (1990) would be to show the following: Each game with an endogenous sharing rule satisfying the assumptions of Simon and Zame (1990) is such that the payoff correspondence has a measurable selection inducing a normalform game whose mixed extension satisfies the assumptions in Reny (1999). We present a result showing that this is not so in general, even not when the assumptions in Reny (1999) are weakened to those in Barelli and Meneghel $(2013)$
\end{abstract}

Journal of Economic Literature Classification Numbers: C72

*We wish to thank Phil Reny, the editor (Françoise Forges) and two anonymous referee for very helpful comments. Financial support from Fundação para a Ciência e Tecnologia (under grant PTDC/EGE-ECO/105415/2008) is gratefully acknowledged. Any remaining errors are, of course, ours.

${ }^{\dagger}$ Address: University of Surrey, Faculty of Business, Economics and Law, School of Economics, Guildford, GU2 7XH, UK; email: g.carmona@surrey.ac.uk.

${ }^{\ddagger}$ Address: Institut für Volkswirtschaftslehre, Universität Wien, Oskar-Morgenstern-Platz 1, A1090 Wien, Austria; email: konrad.podczeck@univie.ac.at 
Keywords: Games with an endogenous sharing rule; discontinuous games; existence of equilibrium.

\section{Introduction}

The approaches of Reny (1999) and of Simon and Zame (1990) both provide mixed strategy equilibrium existence results for general discontinuous games. While the approach of Reny (1999) means to impose certain topological conditions on players' payoff functions in the mixed extension of a given normal form game - summarized by the notion of "better reply security", - the approach of Simon and Zame (1990) replaces the vector of players' payoff function by a correspondence with certain propertiesupper hemicontinuous with nonempty, closed and convex values - and asks whether there is some measurable selection such that the induced normal form game has a mixed strategy equilibrium. As pointed out by Jackson and Swinkels (2005), in some specific contexts such as auction settings, the two approaches seem to be closely related; however, as also pointed out by these authors, "[h]ow these approaches turn out to be related ... is an open question".

In this note we address this question. We first remark that Simon and Zame's (1990) existence result cannot be used to establish the mixed strategy equilibrium existence result of Reny (1999). Indeed, let $\left(X_{i}, u_{i}\right)_{i=1, \ldots, n}$ be a normal form game such that the action sets $X_{i}$ are non-empty compact metric spaces and such that the mixed extension satisfies Reny's condition of better reply security. ${ }^{1}$ Write $X=\prod_{i=1}^{n} X_{i}$, and $u=\left(u_{1}, \ldots, u_{n}\right)$. Then, if one takes for the payoff correspondence $Q: X \rightarrow \mathbb{R}^{n}$ the correspondence defined by setting $Q(x)=\{u(x)\}$ for $x \in X$, Simon and Zame's (1990) existence result does not apply because this latter result requires $Q$ to be closed, which need not be true because better-reply security-regardless of whether in mixed or in pure strategies - does not imply that $u$ is continuous. If one takes for $Q$ the smallest upper hemi-continuous closed- and convex-valued correspondence which includes $u$ (in the sense of set inclusion of the graphs), then Simon and Zame's (1990)

\footnotetext{
${ }^{1}$ See Section 2 for a formal definition.
} 
existence result cannot be used either, just because this result does not control the selection for which there is a mixed strategy equilibrium.

Conversely, with $\left(X_{i}\right)_{i=1, \ldots n}$ and $X$ as above, let $Q: X \rightarrow \mathbb{R}^{n}$ be a payoff correspondence which is upper hemi-continuous with non-empty compact convex values. Then, in order that Reny's (1999) existence result can be used to establish that of Simon and Zame (1990), one would need the existence of a measurable selection of $Q$ that is better-reply secure in mixed strategies.

In this paper we show, however, that in a general game with an endogenous sharing rule the payoff correspondence need not have any measurable selection inducing a normal form game whose mixed extension is better-reply secure. Our result thus suggests that, in general, there is no formal relationship between the existence result of Simon and Zame (1990) and the mixed strategy existence result of Reny (1999).

We also consider the notion of continuous security, which was introduced by Barelli and Meneghel (2013). The mixed strategy version of this notion amounts to a condition that is weaker than the mixed strategy version of better reply security (see Barelli and Meneghel (2013, Proposition 2.4)). Nevertheless, a similar conclusion as with better reply security is true. In fact, we show that the payoff correspondence of a game with an endogenous sharing rule need not have any measurable selection such that the mixed extension of the induced normal form game is continuously secure.

Closing the introduction, we comment on a paper by Bich and Laraki (2017). For a normal form game $\left(X_{i}, u_{i}\right)_{i=1, \ldots, n}$ as above, and $Q: X \rightarrow \mathbb{R}^{n}$ the smallest upper hemicontinuous closed- and convex-valued correspondence which includes $u=\left(u_{1}, \ldots, u_{n}\right)$, Bich and Laraki (2017) showed (see their Remark 4.4) that the method of proof used in Simon and Zame (1990) can be used to prove Reny's (1999) mixed strategy existence result if the mixed extension of the original game $\left(X_{i}, u_{i}\right)_{i=1, \ldots, n}$ is better reply secure. This seems to be the largest extent to which one can relate the work of Simon and Zame (1990) and Reny (1999); indeed, in line with our result, the hypothesis in the existence results for discontinuous games by Reny (1999) and by Simon and Zame (1990) are incomparable. 


\section{The result}

For our purposes, it suffices to consider 2-player games where each player has the closed unit interval $[0,1]$ as his action set. We write $I=\{0,1\}$ for the set of these players. Given $i \in I$, we write $-i$ for $I \backslash\{i\}$. Also, we write $M$ for the set of Borel probability measures on $[0,1]$. Thus $M$ is the set of mixed strategies available for each of the two players. We regard $M$ as being endowed with the narrow topology, ${ }^{2}$ and $M \times M$ with the corresponding product topology.

In the context specified in the previous paragraph, a normal form game is fully determined by a bounded measurable function $u=\left(u_{1}, u_{2}\right):[0,1] \times[0,1] \rightarrow \mathbb{R}^{2}$, with the interpretation that given $x=\left(x_{1}, x_{2}\right) \in[0,1] \times[0,1]$, the payoff of player $i$ is $u_{i}(x), i=1,2$.

Given a normal form game $u:[0,1] \times[0,1] \rightarrow \mathbb{R}^{2}$, the mixed extension of $u$ is the function $\bar{u}=\left(\bar{u}_{1}, \bar{u}_{2}\right): M \times M \rightarrow \mathbb{R}^{2}$ defined by setting $\bar{u}_{i}\left(\sigma_{1}, \sigma_{2}\right)=\int_{[0,1] \times[0,1]} u_{i} \mathrm{~d} \sigma_{1} \times \sigma_{2}$ for $i=1,2$ and $\left(\sigma_{1}, \sigma_{2}\right) \in M \times M$. Thus, given $\left(\sigma_{1}, \sigma_{2}\right) \in M \times M, \bar{u}_{i}\left(\sigma_{1}, \sigma_{2}\right)$ is the expected payoff of player $i$.

A mixed strategy Nash equilibrium of the game $u$ is a pair $\left(\sigma_{1}, \sigma_{2}\right) \in M \times M$ such that $\bar{u}_{i}\left(\sigma_{i}, \sigma_{-i}\right) \geq \bar{u}_{i}\left(\sigma_{i}^{\prime}, \sigma_{-i}\right)$ for each $i \in I$ and each $\sigma_{i}^{\prime} \in M$. Of course, this is equivalent to saying that $\left(\sigma_{1}, \sigma_{2}\right)$ is a pure strategy Nash equilibrium of the mixed extension $\bar{u}$ of $u$.

Let $u$ be a normal form game, with mixed extension $\bar{u}$. We say that $u$ is mixedstrategy-better-reply secure if whenever $\left(\sigma_{1}, \sigma_{2}, r_{1}, r_{2}\right) \in M \times M \times \mathbb{R}^{2}$ belongs to the closure of the graph of $\bar{u}$ and $\left(\sigma_{1}, \sigma_{2}\right)$ is not a mixed strategy Nash equilibrium of $u$, there is an $i \in I$, a number $\alpha>r_{i}$, a $\bar{\sigma}_{i} \in M$, and a neighborhood $V$ of $\sigma_{-i}$ in $M$ such that $\bar{u}_{i}\left(\bar{\sigma}_{i}, \sigma_{-i}^{\prime}\right) \geq \alpha$ for all $\sigma_{-i}^{\prime} \in V$. In other words, this amounts to the requirement that the mixed extension $\bar{u}$ of $u$ be better-reply secure.

We say that $u$ is mixed-strategy-continuously secure if whenever $\left(\sigma_{1}, \sigma_{2}\right) \in M \times M$ is not a mixed strategy Nash equilibrium, there is an $\alpha \in \mathbb{R}^{2}$, a neighborhood $V$ of

\footnotetext{
${ }^{2}$ Recall that the narrow topology on $M$ is the coarsest topology on $M$ making the map $\mu \mapsto \int f \mathrm{~d} \mu$ continuous for every bounded continuous real-valued function $f$ on $[0,1]$. Furthermore, recall that the Prohorov metric metricizes the narrow topology on $M$.
} 
$\left(\sigma_{1}, \sigma_{2}\right)$ in $M \times M$, and a closed nonempty-valued correspondence $\varphi_{i}: V \rightarrow M$ for each $i \in I$ such that

(a) for any $\left(\sigma_{1}^{\prime}, \sigma_{2}^{\prime}\right) \in V$ and each $i \in N, \varphi_{i}\left(\sigma_{1}^{\prime}, \sigma_{2}^{\prime}\right) \subseteq\left\{\gamma_{i} \in M: \bar{u}_{i}\left(\gamma_{i}, \sigma_{-i}^{\prime}\right) \geq \alpha_{i}\right\}$,

(b) for any $\left(\sigma_{1}^{\prime}, \sigma_{2}^{\prime}\right) \in V$, there is an $i \in I$ such that $\sigma_{i}^{\prime} \notin \operatorname{co}\left\{\gamma_{i} \in M: \bar{u}_{i}\left(\gamma_{i}, \sigma_{-i}^{\prime}\right) \geq\right.$ $\left.\alpha_{i}\right\} .^{3}$

In other words, this amounts to the requirement that the mixed extension $\bar{u}$ of $u$ be continuously secure.

Given any bounded measurable function $u:[0,1] \times[0,1] \rightarrow \mathbb{R}^{2}$, we write $Q_{u}$ for the smallest convex-valued and closed correspondence $Q:[0,1] \times[0,1] \rightarrow \mathbb{R}^{2}$ which includes $u$ in the sense of set inclusion of the graphs, i.e. $Q_{u}(x)=\operatorname{co}\left\{r \in \mathbb{R}^{2}:(x, r) \in\right.$ $\left.\Gamma_{u}\right\}$ for each $x \in[0,1] \times[0,1]$, where $\Gamma_{u}$ denotes the closure of the graph of $u$ and co stands for "convex hull". We write $S_{Q_{u}}$ for the set of all measurable selections of $Q_{u}$. Note that boundedness of $u$ implies that $Q_{u}$ must actually be upper hemicontinuous with compact values; in particular, every element of $S_{Q_{u}}$ must be bounded and therefore defines a normal form game in the sense of the above specifications. Here is our result; recall that, as noted in the introduction, mixed-strategy-betterreply security implies mixed-strategy-continuous security.

Theorem. There is a bounded measurable function $u^{*}:[0,1] \times[0,1] \rightarrow \mathbb{R}^{2}$ such that no $u \in S_{Q_{u^{*}}}$ is mixed-strategy-continuously secure; in particular, no $u \in S_{Q_{u^{*}}}$ is mixed-strategy-better-reply secure.

The proof of the theorem is in the next section, which also contains some intuition for it.

\footnotetext{
${ }^{3}$ This definition of continuous security is as in Barelli and Meneghel (2013) but it is not a sufficient condition for the existence of equilibrium. To solve this problem, one can require $\varphi_{i}$ to be convex, as in Barelli and Soza (2009), or to be included in a finite-dimensional subspace of $M$, as in McLennan, Monteiro, and Tourky (2011).
} 


\section{Proof of the theorem}

In the sequel, "equilibrium" means "mixed strategy Nash equilibrium." For each $i \in N$ and each $x_{i} \in[0,1]$, we write $\delta_{x_{i}}$ for the Dirac measure at $x_{i}$. Also, we write $X=[0,1] \times[0,1]$. Let

$$
S_{1}=[2 / 3,1] \times[0,1 / 2] .
$$

Let $A$ denote the set of even natural numbers that are greater or equal than 6 , and for each $n \in A$, let

$$
S_{2}^{n}=\left\{\left(x_{1}, x_{2}\right) \in X: x_{1} \in\left[\frac{1}{n+1}, \frac{1}{n}\right] \text { and } x_{1} \leq x_{2} \leq 2 x_{1}\right\} .
$$

Let $S_{2}=\bigcup_{n \in A} S_{2}^{n}$. Define $u^{*}=\left(u_{1}^{*}, u_{2}^{*}\right): X \rightarrow \mathbb{R}^{2}$ by setting

$$
u^{*}\left(x_{1}, x_{2}\right)=\left\{\begin{array}{l}
(1,1) \text { if }\left(x_{1}, x_{2}\right) \in S_{1} \\
(2,2) \text { if }\left(x_{1}, x_{2}\right) \in S_{2} \\
(0,0) \text { if }\left(x_{1}, x_{2}\right) \in X \backslash\left(S_{1} \cup S_{2}\right) .
\end{array}\right.
$$

Note that $Q_{u^{*}}(0,0)=\{(2 \lambda, 2 \lambda): \lambda \in[0,1]\}, Q_{u^{*}}(1,1)=Q_{u^{*}}(0,1)=\{(0,0)\}$, and $Q_{u^{*}}(1,0)=\{(1,1)\}$.

Fix any $u \in S_{Q_{u^{*}}}$ and recall that $\bar{u}$ denotes the mixed extension of $u$.

To see that $u$ is not mixed-strategy-continuously secure, assume first that $u$ is such that $\left(\delta_{0}, \delta_{1}\right)$ is an equilibrium of $u$. Because $u(0,1)=(0,0)$ and $u(0,0)=(2 \lambda, 2 \lambda)$ for some $\lambda \in[0,1]$, it follows that $u(0,0)=(0,0)$. Since $u(1,0)=(1,1)$, it follows that $\left(\delta_{0}, \delta_{0}\right)$ is not a equilibrium of $u$. Suppose that $u$ is mixed-strategy-continuously secure. Then, in particular, there is an $\alpha \in \mathbb{R}^{2}$, an open neighborhood $V \subseteq M \times M$ of $\left(\delta_{0}, \delta_{0}\right)$, and nonempty-valued closed correspondences $\varphi_{i}: V \rightarrow X_{i}$ for each $i \in I$, such that conditions (a) and (b) in the definition of continuous security hold. Let $\left(x_{1}^{\prime}, x_{2}^{\prime}\right) \in X$ be such that $\left(\delta_{x_{1}^{\prime}}, \delta_{x_{2}^{\prime}}\right) \in V, x_{1}^{\prime}<x_{2}^{\prime}<2 x_{1}^{\prime}$ and $x_{1}^{\prime} \in(1 /(n+1), 1 / n)$ for some $n \in A$. Then $x^{\prime}$ belongs to the interior of $S_{2}^{n}$, so $u\left(x^{\prime}\right)=(2,2)$. Condition (b) in the definition of mixed-strategy-continuous security therefore implies that $\alpha_{i}>2$ for some $i \in I$. This, however, implies that condition (a) in the definition of continuous security cannot hold because $u_{i}\left(\hat{x}_{i}, x_{-i}^{\prime}\right) \leq 2$ for all $\hat{x}_{i} \in[0,1]$ and all $i \in I$. This contradiction shows that $u$ is not mixed-strategy-continuously secure. 
Now assume that $u$ is such that $\left(\delta_{0}, \delta_{1}\right)$ is not an equilibrium of $u$. Again suppose that $u$ is continuously secure. Then there exists $\alpha \in \mathbb{R}^{2}$, an open neighborhood $V \subseteq M \times M$ of $\left(\delta_{0}, \delta_{1}\right)$, and closed nonempty-valued correspondences $\varphi_{i}: V \rightarrow M_{i}$ for each $i \in I$, such that conditions (a) and (b) in the definition of continuous security hold. Let $x^{\prime} \in X$ be such that $\left(\delta_{x_{1}^{\prime}}, \delta_{x_{2}^{\prime}}\right) \in V, x_{1}^{\prime} \in(1 /(n+1), 1 / n)$ for some odd $n \geq 6$ (i.e. $n \in A^{c}$ ), and $x_{2}^{\prime}>1 / 2$. Then $u_{1}\left(y_{1}, x_{2}^{\prime}\right)=0$ for all $y_{1} \in[0,1]$ and $u_{2}\left(x_{1}^{\prime}, y_{2}\right)=0$ for all $y_{2} \in[0,1]$. This, together with condition (a) in the definition of continuous security, implies that $\alpha_{i} \leq 0$ for each $i \in I$. But then $\left\{\gamma_{i} \in M: \bar{u}_{i}\left(\gamma_{i}, \delta_{x_{-i}^{\prime}}\right) \geq \alpha_{i}\right\}=M$ for each $i \in I$, and condition (b) in the definition of continuous security fails. This contradiction shows that $u$ is not mixed-strategy-continuously secure also if $\left(\delta_{0}, \delta_{1}\right)$ is not an equilibrium of $u$.

We conclude this section by noting that a simpler example would suffice to show that no $u \in S_{Q_{u^{*}}}$ is mixed-strategy-better-reply secure. Specifically, modify the functions $u^{*}$ by changing the definition of the set $S_{2}$ to

$$
S_{2}=\left\{\left(x_{1}, x_{2}\right) \in X: x_{1} \leq 1 / 4 \text { and } x_{1} \leq x_{2} \leq 2 x_{1}\right\} .
$$

Some intuition is now as follows. Saying that there is no $u \in S_{Q_{u^{*}}}$ which is mixed-strategy-better-reply secure is the same as saying that neither $u^{*}$ is mixedstrategy-better-reply-secure, nor can $u^{*}$ be changed at its points of discontinuity to obtain a $u$ which is mixed-strategy-better-reply-secure. Perhaps the easiest way to see that $u^{*}$ is not mixed-strategy-better-reply-secure is to consider $\left(\delta_{0}, \delta_{1}\right)$ which is not an equilibrium of $u^{*}\left(\right.$ since $\left.u_{2}^{*}(0,1)=0<2=u_{2}^{*}(0,0)\right)$. The vector $\left(\delta_{0}, \delta_{1}, 0,0\right)$ belongs to the graph of the mixed extension of $u^{*}$, but no player can secure a payoff strictly above 0 at $\left(\delta_{0}, \delta_{1}\right)$. The same argument holds for any $u \in S_{Q_{u^{*}}}$ provided that $\left(\delta_{0}, \delta_{1}\right)$ is not an equilibrium of $u$. This property can fail but only if $u_{2}(0,0)=0$; this, in turn, implies that $u_{1}(0,0)=0$ (as $\left.u_{1}=u_{2}\right)$ and that $\left(\delta_{0}, \delta_{0}\right)$ is not an equilibrium of $u$ (as $\left.u_{1}(1,0)=1\right)$. Since $\left(\delta_{0}, \delta_{0}, 2,2\right)$ belongs to the closure of the graph of the mixed extension of $u$ and the payoff of each player is bounded above by 2 , no player can secure a payoff strictly above 2 at $\left(\delta_{0}, \delta_{0}\right)$. 


\section{References}

Barelli, P., and I. Meneghel (2013): "A Note on the Equilibrium Existence Problem in Discontinuous Games," Econometrica, 81, 813-824.

Barelli, P., And I. Soza (2009): "On the Existence of Nash Equilibria in Discontinuous and Qualitative Games," University of Rochester.

Bich, P., and R. Laraki (2017): "On the Existence of Approximate Equilibria and Sharing Rule Solutions in Discontinuous Games," Theoretical Economics, 12, 79-108.

JaCkson, M., And J. Swinkels (2005): "Existence of Equilibrium in Single and Double Private Value Auctions," Econometrica, 73, 93-139.

Mclennan, A., P. Monteiro, and R. Tourky (2011): "Games with Discontinuous Payoffs: a Strengthening of Reny's Existence Theorem," Econometrica, 79, 1643-1664.

Reny, P. (1999): "On the Existence of Pure and Mixed Strategy Equilibria in Discontinuous Games," Econometrica, 67, 1029-1056.

Simon, L., And W. Zame (1990): "Discontinuous Games and Endogenous Sharing Rules," Econometrica, 58, 861-872. 